\title{
THE LOCAL LIMIT THEOREM AND SOME RELATED ASPECTS OF SUPER-CRITICAL BRANCHING PROCESSES
}

\author{
BY \\ KRISHNA B. ATHREYA( $\left.{ }^{1}\right)$ AND PETER NEY $\left({ }^{2}\right)$
}

\begin{abstract}
Let $\left\{Z_{n}: n=0,1,2, \ldots\right\}$ be a Galton-Watson branching process with offspring p.g.f. $f(s)=\sum_{0}^{\infty} p_{s} s^{\prime}$. Assume (i) $1<m=f^{\prime}(1-)=\sum_{1}^{\infty} j p_{s}<\infty$, (ii) $\sum_{1}^{\infty} j^{2} p_{j}<\infty$ and (iii) $\gamma_{0}=f^{\prime}(q)>0$, where $q$ is the extinction probability of the process. Let $w(x)$ denote the density function of $W$, the almost sure limit of $Z_{n} m^{-n}$ with $Z_{0}=1, w^{(i)}(x)$ the $i$-fold convolution of $w(x), P_{n}(i, j)=P\left(Z_{n}=j \mid Z_{0}=i\right), \delta_{0}=\left(\log \gamma_{0}^{-1}\right)(\log m)^{-1}$ and $\beta_{0}=m^{\delta_{0} /\left(3+\delta_{0}\right)}$. Then for any $0<\beta<\beta_{0}$ and $i$ we can find a constant $C=C(i, \beta)$ such that
\end{abstract}

$$
\left|m^{n} P_{n}(i, j)-w^{(i)}\left(m^{-n} j\right)\right| \leqq C\left[\beta_{0}^{-n}\left(m^{-n} j\right)^{-1}+\beta^{-n}\right]
$$

for all $j \geqq 1$. Applications to the boundary theory of the associated space time process are also discussed.

1. Introduction. Let $\left\{Z_{n}: n=0,1, \ldots\right\}$ be a Galton-Watson (Markov) branching process, with offspring generating function $f(s)=\sum p_{i} s^{i}$. (See [1].) The oldest and most basic limit law in the subject states that there is positive probability of nonextinction if and only if $m=\sum i p_{i}>1$, and that in this case with some additional hypotheses $W_{n}=m^{-n} Z_{n}$ converges to a nondegenerate random variable $W$.

In a series of papers, the hypotheses for this result were weakened, the type of convergence was strengthened, and information was produced about the limit random variable $W$. Hawkins and Ulam [6] proved convergence in distribution when all moments of $\left\{p_{i}\right\}$ existed; Yaglom [15] assumed only a second moment; Harris [4], [5] proved mean square and probability one convergence under a second moment. The observation that $W_{n}$ was a martingale implied probability one convergence assuming no more than the existence of $m$ but said little about $W$.

An important step in this direction was taken by Kesten and Stigum [11], who proved that if $E Z_{1} \log Z_{1}=\infty$ then $P\{W=0\}=1$, while if $E Z_{1} \log Z_{1}<\infty$ then $W$ is nondegenerate, and in fact has an absolutely continuous distribution away from zero. Namely, there is a function $w(x) \geqq 0$ such that

(1) $\lim _{n \rightarrow \infty} P\left\{x_{1}<W_{n} \leqq x_{2} \mid Z_{0}=i\right\}=\int_{x_{1}}^{x_{2}} w^{(i)}(x) d x, \quad 0<x_{1}<x_{2}<\infty$,

Received by the editors November 20, 1969.

AMS 1969 subject classifications. Primary 6067, 6030.

Key words and phrases. Galton-Watson process, branching process, local limit theorems, potential theory, space-time process.

(1) Research sponsored under contract no. DA-31-124-ARO-D-462.

( $\left.{ }^{2}\right)$ Research sponsored by the NIH under grant no. GM 13567.

Copyright (C) 1970, American Mathematical Society 
where $w^{(i)}(x)$ is the $i$-fold convolution of $w(x)$. (This result had : viously been proved by Harris under a second moment. For new, and we believe simpler proofs of the above results, and a complete discussion of this and related topics, we refer the reader to our forthcoming book [1].)

Expressing the "global" limit law (1) in terms of $P_{n}(i, j) \equiv P\left(Z_{n}=j \mid Z_{0}=i\right)$ yields

$$
\lim _{n \rightarrow \infty} \sum_{j=x_{1} m^{n}}^{x_{2} m^{n}} P_{n}(i, j)=\int_{x_{1}}^{x_{2}} w^{(i)}(x) d x=\int_{x_{1} m^{n}}^{x_{2} m^{n}} m^{-n} w^{(i)}\left(y m^{-n}\right) d y,
$$

which suggests that one might expect the "local" law

$$
m^{n} P_{n}(i, j) \sim w^{(i)}\left(j m^{-n}\right) .
$$

A slightly sharper result is in fact true, and we shall prove the following theorem. Let $q$ denote the extinction probability of the process when $P\left(Z_{0}=1\right)=1$. In this $q$ is the smallest nonnegative root of $t=f(t)$. Let $\gamma_{0}=f^{\prime}(q)>0, \delta_{0}=\left(-\log \gamma_{0}\right) / \log m$, $\beta_{0}=m^{\delta_{0} /\left(3+\delta_{0}\right)}$.

THEOREM 1. Let $\left\{Z_{n}: n=0,1,2, \ldots\right\}$ be a supercritical Galton-Watson process with $E\left(Z_{1}^{2} \mid Z_{0}=1\right)<\infty$. Then the following holds for each $i$. Given any $\beta<\beta_{0}$ we can find a constant $C(\beta)$ such that

$$
\left|m^{n} P_{n}(i, j)-w^{(i)}\left(m^{-n} j\right)\right| \leqq C\left[\frac{\beta_{0}^{-n}}{\left(m^{-n} j\right)}+\beta^{-n}\right] \text { for all } j \geqq 1 .
$$

If $E Z_{1} \log Z_{1}=\infty$ but $E Z_{1}<\infty$ then E. Seneta [12] has shown that there exists a sequence $c_{n}$ of norming constants such that $Z_{n} / c_{n}$ converges (in distribution) to a nondegenerate random variable. It is not known, however, whether this limit variable has an absolutely continuous distribution. Although limit theorems for $P_{n}(i, j)$ for $i, j$ fixed, $n \rightarrow \infty$, are known (see e.g. [1], [12]) without moment assumptions, results of the form of Theorem 1 , which are uniform in $j$, are not known when $E Z_{1} \log Z_{1}=\infty$.

The theorem has a somewhat confused history. The first published work on local limit theorems for branching processes was by Chistyakov [2], who claimed a weaker result than (3); namely that for constants $0<c_{1}<c_{2}<\infty$

$$
\lim _{n \rightarrow \infty} \sup _{c_{1} \leqq j m-n \leqq c_{2}}\left|m^{n} P_{n}(1, j)-w\left(m^{-n} j\right)\right|=0 .
$$

He concerned himself primarily with the continuous time Markov branching process (hereafter referred to as the continuous case) and treated both the critical $(m=1)$ and supercritical $(m>1)$ processes. The former contained a gap, in that his estimate (20) was unfounded. This theorem and its application to the study of stationary measures and harmonic functions has since been treated by Kesten, Ney, and Spitzer [10]. Chistyakov repeated his error in the supercritical case, where his estimate on the characteristic function of the process (preceding his expression (32)) is incorrect. (He corrected both errors in $A$ letter to the editor, 
Theor. Verojatnost. i Primenen. 10 (1965), 597-598. (Russian) MR 32 \#6556.) He concluded his paper by giving the results for the discrete case, and stating that the proof was similar to the continuous one.

Recently there has appeared a paper by H. Imai [7], claiming proof of (3) with an additional hypothesis, namely $j$ and $n$ vary in such a way that, for some $0<c_{1}<c_{2}<\infty$,

$$
c_{1} \leqq j m^{-n} \leqq c_{2} .
$$

He repeats identically the error of Chistyakov, his Corollary 2 being wrong. It is easy to construct a counterexample to this corollary by looking at the linear fractional generating function. More strongly, however, we will show after Lemma 2 in $\$ 2$ that this bound can never hold.

The first complete proof of a supercritical local limit theorem is due to $\mathbf{S}$. Karlin [8] who treated the continuous case in an unpublished manuscript $\left({ }^{3}\right)$. He proved the following:

Let $\left\{Z_{t}: t \geqq 0\right\}$ be a continuous time supercritical simple Markov branching process with offspring p.g.f. $h(s)$ and lifetime parameter $\lambda$. Let $a=\lambda\left(h^{\prime}(1)-1\right)$, $h(0)=0, h^{\prime \prime}(1-)<\infty$. Then

$$
P(Z(t)=n \mid Z(0)=1)=e^{-a t}\left[w\left(n e^{-a t}\right)+o(1)\right]
$$

as $n, t \rightarrow \infty$, where $o(1)$ is uniform for $n$ and $t$ increasing to $\infty$ and obeying the constraints $0<c_{1} \leqq n e^{-a t} \leqq c_{2}<\infty$ for some constants $c_{1}$ and $c_{2}$; and where $w(x)$ is the probability density function of the random variable $W=\lim _{t \rightarrow \infty} Z(t) e^{-a t}$.

Karlin's proof uses the Wiener Tauberian theorem, and methods which are particular to the continuous case and do not seem to work easily in the discrete case.

On the other hand our proof for the discrete case works just as well for the continuous case; and hence Theorem 1 also produces a sharper form of the latter result.

There are also some other consequences. One of these is the following sharper form of the global limit law:

THEOREM 2. Let $\left\{Z_{n}: n=0,1,2, \ldots\right\}$ be a supercritical Galton-Watson process with $E\left(Z_{1}^{2}\right)<\infty$ and $P\left(Z_{0}=r\right)=1$ for some $r$. Then for any $\beta<\beta_{0}=m^{\delta_{0} /\left(3+\delta_{0}\right)}$ and fixed $0<x_{1}<x_{2}<\infty$,

$$
\lim _{n \rightarrow \infty} \beta^{n}\left|P\left\{x_{1}<W_{n} \leqq x_{2}\right\}-P\left\{x_{1}<W \leqq x_{2}\right\}\right|=0
$$

where $W_{n}=Z_{n} / m^{n}$ and $W=\lim _{n} W_{n}$.

The proof of Theorem 1 is in $\$ 4$. In $\$ \$ 2$ and 3 we give some lemmas on the characteristic functions of $W$ and $W_{n}$ which are of some interest in their own right.

$\left.{ }^{3}\right)$ We would like to express our thanks to him for making his manuscript available to us. 
$\$ 5$ contains further results on $W$; namely the Lipschitz continuity and strict positivity of $w(\cdot)$, and the global limit Theorem 2 . (Without the positivity of $w(\cdot)$ the local limit theorem would lose much of its punch, since it would then not be clear that $m^{n}$ is always the right norming sequence.) Some partial results on the behavior of $w(\cdot)$ near zero, and on the space-time boundary of the process, are in $§ 6$.

One final point. For convenience we shall carry out the entire analysis under the assumption that $f(0)=0$, hence that the extinction probability $q=0$. The reader will easily convince himself that all lemmas and arguments have their appropriate analogs when $q>0$.

2. Estimates on the characteristic function of $W$. Let $f_{n}=$ the n-fold iterate of $f$. We shall use $c$ 's and $C$ 's, with or without subscripts to denote constants, not necessarily the same ones each time they appear.

The following result is known (see [1], [5]).

LEMMA 1. For any complex $s,|s|<1$,

$$
\lim _{n \rightarrow \infty} \gamma_{0}^{-n} f_{n}(s) \equiv A(s)
$$

exists (recall $\left.\gamma_{0}=f^{\prime}(q)\right)$ and is the unique solution of the functional equation,

$$
A[f(s)]=A(s), \quad|s| \leqq 1,
$$

with boundary conditions $A(0)=0, A^{\prime}(0)=1$. Further for $0 \leqq s<1$,

$$
\gamma_{0}^{-n} f_{n}(s) \nearrow A(s)<\infty, \quad A^{\prime}(s)>0, \quad A(1-)=\infty .
$$

Let $\varphi(u)=E e^{i u w}, \varphi_{n}(u)=E e^{i u w_{n}}$, and $\delta_{0}=\left(\log \gamma_{0}^{-1}\right)(\log m)^{-1}$.

LEMMA 2. If $E Z_{1} \log Z_{1}<\infty$, then

(i) $\lim _{k \rightarrow \infty} m^{\delta_{0} k} \varphi\left(m^{k} u\right)=A[\varphi(u)], u \neq 0$, real;

(ii) $\sup _{-\infty<v<\infty}|v|^{\delta_{0}}|\varphi(v)|<\infty$;

(iii) $\lim _{k \rightarrow \infty} m^{\left(1+\delta_{0}\right) k} \varphi^{\prime}\left(m^{k} u\right)=\varphi^{\prime}(u) A^{\prime}(\varphi(u)), u \neq 0$;

(iv) $\sup _{-\infty<v<\infty}|v|^{1+\delta_{0}}\left|\varphi^{\prime}(v)\right|<\infty$.

Proof. (i) It is well known [5] that $\varphi(u)$ satisfies $\varphi(m u)=f[\varphi(u)]$, which, when iterated, yields

$$
\varphi\left(m^{k} u\right)=f_{k}[\varphi(u)]
$$

Since $m^{-\delta_{0}}=\gamma_{0}$, and $|\varphi(u)|<1$ for $u \neq 0$ (due to nondegeneracy of $W$, see [13, Lemma 1]), our Lemma 1 implies

$$
m^{\delta_{0} k} \varphi\left(m^{k} u\right)=f_{k}[\varphi(u)] / m^{-\delta_{0} k}=f_{k}[\varphi(u)] / \gamma_{0}^{k} \rightarrow A[\varphi(u)] \text { as } k \rightarrow \infty .
$$

(ii) $\left(m^{k}|u|\right)^{\delta_{0}}\left|\varphi\left(m^{k} u\right)\right| \leqq|u|^{\delta_{0}} f_{k}(|\varphi(u)|) / \gamma_{0}^{k} \leqq|u|^{\delta_{0}} A(|\varphi(u)|)$, since $\gamma_{0}^{-k} f_{k}(x) \nearrow A(x)$ for $0<x<1$. Let $\beta=\sup _{1 \leqq|u| \leqq m}|\varphi(u)|$. Then by continuity of $\varphi, \beta<1$. Thus 


$$
\sup _{\substack{1 \leqq|u| \leq m \\ 0 \leqq k}}\left(m^{k}|u|\right)^{\delta_{0}}\left|\varphi\left(m^{k} u\right)\right| \leqq \sup _{1 \leqq|u| \leqq m}|u|^{\delta_{0}} A(|\varphi(u)|) \leqq m^{\delta} 0 A(\beta)<\infty,
$$

or

$$
\sup _{|v| \geqq 1}|v|^{\delta_{0}} \varphi(|v|)<\infty .
$$

Clearly $\sup _{|v| \leqq 1}|v|^{\delta_{0}} \varphi(|v|)<\infty$.

(iii) Differentiating both sides of (3) with respect to $u$ yields

$$
m^{k} \varphi^{\prime}\left(m^{k} u\right)=\varphi^{\prime}(u) f_{k}^{\prime}(\varphi(u))
$$

and hence as $k \rightarrow \infty$

$$
\left(m^{k}\right)^{1+\delta_{0}} \varphi^{\prime}\left(m^{k} u\right)=\left(f_{k}^{\prime}(\varphi(u)) / \gamma_{0}^{k}\right) \varphi^{\prime}(u) \rightarrow A^{\prime}[\varphi(u)] \varphi^{\prime}(u), \quad u \neq 0 .
$$

(iv) From the above identity (iii)

$$
\begin{aligned}
\left(m^{k}|u|\right)^{1+\delta_{0}}\left|\varphi^{\prime}\left(m^{k} u\right)\right| & \leqq|u|^{1+\delta_{0}}\left|\varphi^{\prime}(u)\right| f_{k}^{\prime}(|\varphi(u)|) / \gamma_{0}^{k} \\
& \leqq|u|^{1+\delta_{0}}\left|\varphi^{\prime}(u)\right| A^{\prime}(|\varphi(u)|)
\end{aligned}
$$

due to the monotonicity of $f_{k}^{\prime} / \gamma_{0}^{k}$. Hence

$$
\sup _{1 \leqq \leqq u \mid \leq m}\left[\left(m^{k}|u|\right)^{1+\delta_{0}}\left|\varphi^{\prime}\left(m^{k} u\right)\right|\right] \leqq m^{1+\delta_{0}} A^{\prime}(\beta)<\infty .
$$

Since $\sup _{|v| \leqq 1}|v|^{1+\delta_{0}}\left|\varphi^{\prime}(v)\right|<\infty$, the lemma is proved.

REMARK. We shall now show that Corollary 2 of [7] is incorrect. In our notation the latter asserts that there exists $T>0$ and $c>0$ such that for any $n$

$$
\left|\varphi_{n}(u)\right| \leqq \exp \left(-c u^{2}\right) \text { for }|u|>T
$$

or equivalently $\exp \left(c u^{2}\right)\left|\varphi_{n}(u)\right| \leqq 1$ for $|u|>T$. Since $\varphi_{n}(u) \rightarrow \varphi(u)$ for each $u\left(^{*}\right)$ implies

$$
\exp \left(c u^{2}\right)|\varphi(u)| \leqq 1 \text { for }|u|>T .
$$

Clearly there exists a $\theta>0$ such that $A(\varphi(\theta)) \neq 0$. For this $\theta$ by Lemma 2 (i) we know

$$
\lim _{k \rightarrow \infty}\left(m^{k} \theta\right)^{\delta_{0}}\left|\varphi\left(m^{k} \theta\right)\right|=\theta^{\delta_{0}}|A(\varphi(\theta))|>0 .
$$

On the other hand

$$
\lim _{k \rightarrow \infty}\left(m^{k} \theta\right)^{-\delta_{0}} \exp \left(c\left(m^{k} \theta\right)^{2}\right)=\infty
$$

thus making $\left(^{* *}\right)$ and hence $(*)$ false.

LEMMA 3. If $E Z_{1} \log Z_{1}<\infty$, then there exists a constant $c<\infty$, such that for all $T \geqq 0$

$$
T^{\delta} \sup _{x \geqq 0}\left|x w(x)-\frac{x}{2 \pi} \int_{|u| \leqq T} e^{-i u x} \varphi(u) d u\right| \leqq c .
$$


Proof. Writing $\varphi(u)$ in its integral form and differentiating with respect to $u$ yields

$$
\frac{1}{i} \varphi^{\prime}(u)=\int_{0}^{\infty} e^{i u x} x w(x) d x .
$$

Note that since $E\left(W \mid Z_{0}=1\right)=1, x w(x)$ is a probability density function and also that $\left|\varphi^{\prime}(u)\right|$ is integrable by Lemma 2 (iv). Thus we may apply the usual inversion formula to (5) and get

$$
x w(x)=\frac{1}{2 \pi i} \int_{-\infty}^{\infty} e^{-i u x} \varphi^{\prime}(u) d u=\frac{1}{2 \pi i} \int_{|u| \leqq T} e^{-i u x} \varphi^{\prime}(u) d u+\frac{1}{2 \pi i} \int_{|u|>T} .
$$

Integrating the first integral on the right side by parts yields

$$
\frac{1}{2 \pi i} \int_{|u| \leqq T} e^{-i u x} \varphi^{\prime}(u) d u=\left.\frac{1}{2 \pi i} e^{-i u x} \varphi(u)\right|_{-T} ^{T}+\frac{1}{2 \pi} \int_{-T}^{T} \varphi(u) x e^{-i u x} d u,
$$

which, substituted in (6), implies

$$
x w(x)-\frac{x}{2 \pi} \int_{-T}^{T} \varphi(u) e^{-i u x} d u=\frac{1}{2 \pi i}\left\{e^{-i x T} \varphi(T)-e^{i x T} \varphi(-T)\right\}+\frac{1}{2 \pi i} \int_{|u|>T} \varphi^{\prime}(u) e^{-i u x} d u
$$

and hence

$$
\begin{aligned}
T^{\delta_{0}} x\left|w(x)-\frac{1}{2 \pi} \int_{-T}^{T} \varphi(u) e^{-i u x} d u\right| \leqq & T^{\delta_{0}}|\varphi(T)|+T^{\delta_{0}}|\varphi(-T)| \\
& +T^{\delta_{0}} \int_{|u|>T}\left|\varphi^{\prime}(u)\right| \cdot|u|^{1+\delta_{0}} \frac{d u}{|u|^{1+\delta_{0}}} .
\end{aligned}
$$

Applying Lemma 2 (ii) and (iv) to the right side of this inequality, we see that it is bounded, implying the lemma.

3. Estimates on $W_{n}$.

Lemma 4. $\left|\varphi_{n}(v)-\varphi(v)\right| \leqq v^{2} E\left(W_{n}-W\right)^{2} / 2$.

Proof.

$$
\begin{aligned}
\varphi_{n}(v)-\varphi(v)= & E\left(\exp \left(i v W_{n}\right)-\exp (i v W)\right. \\
= & E\left(\exp \left(i v W_{n}\right)\left[1+i v\left(W-W_{n}\right)-\exp \left(i v\left(W-W_{n}\right)\right)\right]\right) \\
& +i v E\left(\exp \left(i v W_{n}\right)\left(W-W_{n}\right)\right) .
\end{aligned}
$$

The second term on the right side above is zero since $W_{n}$ is a martingale. For the first term, note that for any real $x$

$$
\left|e^{i x}-1-i x\right|=\left|-\int_{0}^{x} \int_{0}^{t} e^{i u} d u d t\right| \leqq x^{2} / 2
$$

and the lemma follows.

Lemma 5. Assume that $E Z_{1} \log Z_{1}<\infty$. Let

$$
d(K, n)=\sup \left\{\left|f_{n}\left(e^{i u}\right)\right|: K m^{-n-1} \leqq|u| \leqq K m^{-n}\right\} .
$$

Given any $\eta>0$, there exist $K(\eta), N(\eta)<\infty$ such that $d(K, n)<\eta$ for all $n \geqq N$. 
Proof. Since $\varphi_{n}(u)=f_{n}\left(\exp \left(\right.\right.$ ium $\left.\left.^{-n}\right)\right)$,

$$
d(K, n)=\sup \left\{\left|\varphi_{n}(v)\right|: K m^{-1} \leqq|v| \leqq K\right\} .
$$

By Lemma 2 (ii) there exists a constant $C$ such that

$$
\sup \left\{|\varphi(v)|: K m^{-1} \leqq|v| \leqq K\right\} \leqq C\left(K m^{-1}\right)^{-\delta_{0}} .
$$

Given $\eta>0$ choose $K=K(\eta)$ such that

$$
C\left(K m^{-1}\right)^{-\delta_{0}}<\eta / 2 .
$$

Since $\varphi_{n}(u) \rightarrow \varphi(u)$ uniformly on compact sets there exists a $N=N(\eta)$ such that $n \geqq N$ implies

$$
\sup _{K m^{-1} \leqq|v| \leqq K}\left|\varphi_{n}(v)-\varphi(v)\right| \leqq \eta / 2
$$

with $K$ as chosen in (3). Then (2) and (4) imply the lemma.

Lemma 6. Assume $E Z_{1} \log Z_{1}<\infty$. Let

$$
a(K, n)=\sup \left\{\left|f_{n}\left(e^{i u}\right)\right|: K m^{-n} \leqq|u| \leqq \pi\right\} .
$$

Given any $\eta>0$, there exist $K, N<\infty$ such that $a(K, n)<\eta$ for all $n>N$.

Proof. We have $f_{n}\left(e^{i u}\right)=f\left(f_{n-1}\left(e^{i u}\right)\right),\left|f_{n}\left(e^{i u}\right)\right| \leqq f\left(\left|f_{n-1}\left(e^{i u}\right)\right|\right)$, and hence $\sup \left\{\left|f_{n}\left(e^{i u}\right)\right|: K m^{-n} \leqq|u| \leqq \pi\right\}$

$$
\begin{aligned}
& \leqq f\left(\sup \left\{\left|f_{n-1}\left(e^{i u}\right)\right|: K m^{-n} \leqq|u| \leqq \pi\right\}\right) \\
& =f\left[\operatorname { m a x } \left[\sup \left\{f_{n-1}\left(e^{i u}\right): K m^{-n} \leqq|u| \leqq K m^{-(n-1)}\right\},\right.\right. \\
& \left.\left.\quad \sup \left\{f_{n-1}\left(e^{i u}\right): K m^{-(n-1)} \leqq|u| \leqq \pi\right\}\right]\right],
\end{aligned}
$$

i.e.

$$
a_{K, n} \leqq f\left[d_{K, n-1} \vee a_{K, n-1}\right] \quad \text { (where } a \vee b \text { means } \max (a, b) \text { ). }
$$

Now choose $N, K$ as in Lemma 5 . Then

$$
d_{K, n-1}<\eta \text { for } n>N
$$

and hence

$$
a_{K, n} \leqq f\left(\eta \vee a_{K, n-1}\right) \text { for all } n \geqq N .
$$

If $a_{K, N} \leqq \eta$ then by (8), $a_{K, N+1} \leqq f(\eta) \leqq \eta$ and hence

$$
a_{K, n} \leqq \eta \quad \text { for all } n \geqq N .
$$

On the other hand, if $a_{K, N}>\eta$ then, by (8), $a_{K, N+1} \leqq f\left(a_{K, N}\right)$ and iterating

$$
a_{K, N+j} \leqq f_{j}\left(a_{K, N}\right)
$$

as long as $a_{K, N+j}>\eta$. But the right side of (10) goes to zero as $j \rightarrow \infty$, and hence the left must eventually be $\leqq \eta$. By (9) it then remains $\leqq \eta$. This proves Lemma 6 . 
4. Proof of the main result. We restate Theorem 1 in the form in which we shall here prove it. Since the proof for a general $i$ is identical to the case of $i=1$ we shall prove instead of (3) of $\$ 1$ the following version.

TheORem 1a. Let $P_{n}(i, j)$ be the transition function of a Galton-Watson process with $m>1, q=0$, and $E Z_{1}^{2}<\infty$. Then the following holds:

Given any $\beta<\beta_{0}=m^{\delta_{0} /\left(3+\delta_{0}\right)}$ one can find a constant $C(\beta)$ such that for any $j \geqq 1$

$$
\left|m^{n} P_{n}(1, j)-w\left(m^{-n} j\right)\right| \leqq C\left[\beta_{0}^{-n} /\left(m^{-n} j\right)+\beta^{-n}\right] .
$$

Proof. Noting that $f_{n}\left(e^{i u}\right)=\sum_{r=1}^{\infty} P_{n}(1, r) e^{i r u}$ for $-\pi \leqq u \leqq \pi$ we have

$$
\left|m^{n} P_{n}(1, j)-w\left(m^{-n} j\right)\right|=\left|m^{n} \frac{1}{2 \pi} \int_{0 \leqq|u| \leqq \pi} f_{n}\left(e^{i u}\right) e^{-i j u} d u-w\left(m^{-n} j\right)\right|
$$

On adding and subtracting $1 / 2 \pi \int_{|u| \leqq m^{(1-\alpha) n}} \exp \left(-i u\left(j m^{-n}\right)\right) \varphi(u) d u$ (where $0<\alpha<1$ is to be specified later) to the right side of (1), it is

$$
\begin{aligned}
\leqq & \left|m^{n} \frac{1}{2 \pi} \int_{0 \leqq|u| \leqq \pi} f_{n}\left(e^{i u}\right) e^{-i j u} d u-\frac{1}{2 \pi} \int_{|u| \leqq m^{(1-\alpha) n}} \exp \left(-i u\left(j m^{-n}\right)\right) \varphi(u) d u\right| \\
& +\left|w\left(m^{-n} j\right)-\frac{1}{2 \pi} \int_{|u| \leqq m^{(1-\alpha) n}} \exp \left(-i u\left(j m^{-n}\right)\right) \varphi(u) d u\right| .
\end{aligned}
$$

Denote the first term on the right side of (2) by $\mathrm{I}^{(n)}$ and the second by $\mathrm{II}^{(n)}$.

Applying Lemma 3 to $\mathrm{II}^{(n)}$ with $x=m^{-n} j$, and $T=m^{(1-\alpha) n}$, we see that there is a constant $C$ such that

$$
m^{(1-\alpha) n \delta_{0}} \mathrm{II}^{(n)} \leqq C\left(m^{-n} j\right)^{-1},
$$

i.e.

$$
\mathrm{II}^{(n)} \leqq C m^{-(1-\alpha) n \delta_{0}}\left(m^{-n} j\right)^{-1} .
$$

Thus if we take $\alpha<1$ then $\left(m^{-n} j\right) \mathrm{II}^{(n)}$ decreases geometrically. Next, we decompose $I^{(n)}$ into

(4)

$$
\begin{aligned}
I^{(n)}= & \left|m^{n} \frac{1}{2 \pi} \int_{0 \leqq|u| \leqq \pi} f_{n}\left(e^{i u}\right) e^{-i j u} d u-\frac{1}{2 \pi} \int_{|u| \leqq m^{(1-\alpha) n}} \exp \left(-i u\left(j m^{-n}\right)\right) \varphi(u) d u\right| \\
\leqq & \left(j m^{-n}\right)^{-1}\left|j \int_{m^{-\alpha n} \leqq|u| \leqq \pi} f_{n}\left(e^{i u}\right) e^{-i j u} d u\right| \\
& +\mid m^{n} \frac{1}{2 \pi} \int_{0 \leqq|u| \leqq m^{-\alpha n}} f_{n}\left(e^{i u}\right) e^{-i j u} d u \\
& -\frac{1}{2 \pi} \int_{|u| \leqq m^{(1-\alpha) n}} \exp \left(-i u\left(j m^{-n}\right)\right) \varphi(u) d u \mid .
\end{aligned}
$$

Denote the first term in (4) by $I_{1}^{(n)}$ and the second by $I_{2}^{(n)}$. Consider $I_{2}^{(n)}$ first. In the first integral in $\mathrm{I}_{2}^{(n)}$ make the change of variable $u \rightarrow u m^{-n}$. Then

$$
\begin{aligned}
m^{n} \frac{1}{2 \pi} \int_{0 \leqq|u| \leqq m-\alpha n} & f_{n}\left(e^{i u}\right) e^{-i j u} d u \\
& =\frac{1}{2 \pi} \int_{0 \leqq|u| \leqq m^{(1-\alpha) n}} f_{n}\left(\exp \left(i u m^{-n}\right)\right) \exp \left(-i j u m^{-n}\right) d u,
\end{aligned}
$$


and

$$
\mathbf{I}_{2}^{(n)}=\mid \frac{1}{2 \pi} \int_{0 \leqq|u| \leqq m^{(1-\alpha) n}}\left\{f_{n}\left(\exp \left(\text { ium }^{-n}\right)\right)-\varphi(u)\right\} \exp \left(-i j u m^{-n}\right) d u \mid
$$

and hence

$$
\mathrm{I}_{2}^{(n)} \leqq C \int_{0 \leqq|u| \leqq m^{(1-\alpha) n}} \mid f_{n}\left(\exp \left(\text { ium }^{-n}\right)\right)-\varphi(u) \mid d u .
$$

To estimate the latter we use Lemma 4. Recall that $\varphi_{n}(v)=E \exp \left(i v W_{n}\right)=$ $E \exp \left(i v m^{-n}\right) Z_{n}=f_{n}\left(\exp \left(i v m^{-n}\right)\right)$ and $E\left(W_{n}-W\right)^{2} \leqq c_{1} m^{-n}$. Hence (5) implies

$$
\mathrm{I}_{2}^{(n)} \leqq \int_{0 \leqq|u| \leqq m^{(1-\alpha) n}} c_{2} u^{2} m^{-n} d u \leqq c_{3} m^{3(1-\alpha) n-n},
$$

or

$$
\mathrm{I}_{2}^{(n)} \leqq c\left[m^{(2-3 \alpha)}\right]^{n}
$$

If $\alpha>\frac{2}{3}$ then $I_{2}^{(n)} \searrow 0$ geometrically. Thus we now have the restriction

$$
\frac{2}{3}<\alpha<1 \text {. }
$$

It thus remains to estimate

$$
\mathrm{I}_{1}^{(n)}=\left(j m^{-n}\right)^{-1}\left|j \int_{m^{-\alpha n} \leqq|u| \leqq \pi} f_{n}\left(e^{i u}\right) e^{-i j u} d u\right|
$$

To this end we will use Lemma 6. First, integrating (8) by parts yields

$$
\left(j m^{2}\right) I_{1}^{(n)}=\left|I_{11}^{(n)}+I_{12}^{(n)}\right|,
$$

where

and

$$
\mathrm{I}_{11}^{(n)}=-\left.(1 / i) f_{n}\left(e^{i u}\right) e^{-i j u}\right|_{m^{-\alpha n} \leqq|u| \leqq \pi}
$$

$$
\mathrm{I}_{12}^{(n)}=\int_{m^{-\alpha n} \leqq|u| \leqq \pi} f_{n}^{\prime}\left(e^{i u}\right) e^{-i j u} e^{i u} d u .
$$

Now since $e^{-i j \pi} f_{n}\left(e^{i \pi}\right)=e^{i j \pi} f_{n}\left(e^{-i \pi}\right)$,

$$
I_{11}^{(n)}=-(1 / i)\left[f_{n}\left(\exp \left(-i m^{-\alpha n}\right)\right) \exp \left(i j m^{-\alpha n}\right)-f_{n}\left(\exp \left(i m^{-\alpha n}\right)\right) \exp \left(-i j m^{-\alpha n}\right)\right] .
$$

Furthermore

$$
f_{n}\left(\exp \left(-i m^{-\alpha n}\right)\right)=f_{n-k_{n}}\left(f_{k_{n}}\left(\exp \left(-i m^{-\alpha n}\right)\right)\right)
$$

where $k_{n}=[\alpha n]$, and

$$
f_{k_{n}}\left(\exp \left(-i m^{-\alpha n}\right)\right)=\varphi_{k_{n}}\left(m^{-\alpha n+k_{n}}\right) .
$$

Since $\varphi_{r}(u) \rightarrow \varphi(u)$ and $|\varphi(u)|<1$ for $u \neq 0$ there exist $\beta$ and $r$ such that $r \geqq r_{0}$ implies

$$
\sup _{m-1 \leqq|u| \leqq 1}\left|\varphi_{r}(u)\right| \leqq \beta<1 .
$$


Thus for $n$ such that $[\alpha n] \geqq r_{0}$

$$
\left|f_{n}\left(e^{ \pm i m-\alpha n}\right)\right| \leqq f_{n-k_{n}}(\beta)
$$

and hence by Lemma 1

$$
\leqq \text { const } \gamma_{0}^{n-k_{n}} \leqq \text { const } \gamma_{0}^{n(1-\alpha)} \text {. }
$$

Finally we turn to $I_{12}^{(n)}$. We consider the positive range of $u$, namely

$$
\mathrm{I}_{12}^{(n)+}=\int_{m^{-\alpha n}}^{\pi} f_{n}^{\prime}\left(e^{i u}\right) e^{-i j u} d u,
$$

the integral $\mathrm{I}_{12}^{(n)-}$ over negative $u$ 's being treated similarly. Note the identity

$$
f_{n}^{\prime}\left(e^{i u}\right)=\prod_{j=0}^{n-1} f^{\prime}\left[f_{j}\left(e^{i u}\right)\right]
$$

The idea now is to break the product (12) into two parts

$$
f_{n}^{\prime}\left(e^{i u}\right)=\prod_{j=0}^{J(u, n)} f^{\prime}\left[f_{j}\left(e^{i u}\right)\right] \prod_{j=J(u, n)+1}^{n-1} f^{\prime}\left[f_{j}\left(e^{i u}\right)\right] \equiv P_{n}(u) \cdot Q_{n}(u),
$$

and to choose $J(u, n)$ in such a way that

(i) $\left|f^{\prime}\left(f_{j}\left(e^{i u}\right)\right)\right| \leqq \gamma$ for $j \geqq J(u, n)$,

where $\gamma=f^{\prime}(\eta) \in\left(\gamma_{0}, 1\right)$ can be taken arbitrarily close to $\gamma_{0}$ by choosing $\eta$ sufficiently small;

(ii) $\left|f^{\prime}\left(f_{j}\left(e^{i u}\right)\right)\right| \leqq m$ for $j<J(u, n)$

(this inequality is trivially true);

(iii) $\gamma^{n-J(u, n)} m^{J(u, n)}$ is integrable in $u$.

To achieve (i) we want to take $J$ large, while for (iii) we want $J$ small. The right balance is obtained as follows:

(a) Pick $\gamma=f^{\prime}(\eta)$ arbitrarily close to $\gamma_{0}$ by taking $\eta$ sufficiently small $\left(f^{\prime}(q)=\gamma_{0}\right)$.

(b) With this $\eta$ choose $K_{0}, N_{0}$, satisfying the conditions of Lemma 6.

(c) Choose $k_{0}$ such that $m^{k_{0}}>K_{0}$, and $k_{0}>N_{0}+\log \pi / \log m$.

(d) Choose $n \geqq k_{0} /(1-\alpha) \vee N_{0}$.

For any $u$ in $\left[m^{-\alpha n}, \pi\right]$ define $\theta(u, n)$ by $u=m^{-n \theta(u, n)}$, and take

$$
J(u, n)=[\theta n]+k_{0}=[-\log u / \log m]+k_{0}
$$

where $[x]=($ the largest integer in $|x|)(\operatorname{sgn} x)$. Now due to condition (c), $j>N_{0}$ for all terms in this product. Furthermore (c) and $j>-\log u / \log m+k_{0}$ imply $K_{0} m^{-j} \leqq u$. Hence

$$
\left|Q_{n}(u)\right| \leqq \prod_{j=J+1}^{n-1} f^{\prime}\left\{\sup _{K_{0} m-j \leqq v \leqq \pi}\left|f_{j}\left(e^{i v}\right)\right|\right\}=\prod_{j=J+1}^{n-1} f^{\prime}\left(a\left(K_{0}, j\right)\right)
$$

which by Lemma 6 yields

$$
\left|Q_{n}(u)\right| \leqq \prod_{j=J+1}^{n-1} f^{\prime}(\eta)=\gamma^{n-1-J-1}=\gamma^{n-[\theta n]-k_{0}},
$$


and finally

$$
\left|Q_{n}(u)\right| \leqq c \gamma^{(1-\theta) n} .
$$

(Note that (d) guarantees $n-1 \geqq J+1$.)

Turning to $P_{n}(u)$ we note that this product has $J(u, n)$ terms and use the gross estimate

$$
\left|P_{n}(u)\right| \leqq c m^{\theta n}
$$

Combining (17) and (18) we see that for $m^{-\alpha n} \leqq|u| \leqq \pi$

$$
\left|f_{n}^{\prime}\left(e^{i u}\right)\right| \leqq c\left(m^{\theta} \gamma^{1-\theta}\right)^{n}
$$

Although this bound (and in fact $f_{n}^{\prime}$ itself) need not be bounded, we shall sis that it is integrable. In fact for $0<p<1$ (19) yields

$$
|u|^{p}\left|f_{n}^{\prime}\left(e^{i u}\right)\right| \leqq c\left[m^{\theta(1-p)} \gamma^{1-\theta}\right]^{n}
$$

But $m^{\theta(1-p)} \gamma^{(1-\theta)}$ is increasing in $\theta$, and $u \geqq m^{-\alpha n}$ implies $\theta \leqq \alpha$. Hence the abivvi inequality yields

$$
\sup \left\{|u|^{p}\left|f_{n}^{\prime}\left(e^{i u}\right)\right|: m^{-\alpha n} \leqq u \leqq \pi\right\} \leqq c\left(\gamma^{(1-\alpha)} m^{\alpha(1-p)}\right)^{n} .
$$

So far our only restriction on $\alpha$ is $\frac{2}{3}<\alpha<1$. We may now take $p$ sufficiently close to 1 so that

$$
\gamma^{1-\alpha} m^{\alpha(1-p)}<1
$$

Then

$$
\begin{aligned}
\left|\mathbf{I}_{12}^{(n)+}\right| & \leqq \int_{m^{-\alpha n} \leqq u \leqq \pi}\left|f_{n}^{\prime}\left(e^{i u}\right)\right| d u \leqq \int \frac{|u|^{p}\left|f_{n}^{\prime}\left(e^{i u}\right)\right|}{|u|^{p}} d u \\
& \leqq c\left(\gamma^{(1-\alpha)} m^{\alpha(1-p)}\right)^{n} \int_{m^{-\alpha n}}^{\pi} \frac{d u}{|u|^{p}}
\end{aligned}
$$

The last integral converges, and combining the above with a similar bound for $\mathrm{I}_{12}^{(n)}$ - we get

$$
\left|\mathrm{I}_{12}^{(n)}\right| \leqq c_{1}\left[\gamma^{(1-\alpha)} m^{\alpha(1-p)}\right]^{n} .
$$

Summarizing the estimates in (3), (6), (10), (21) we see that

$$
\begin{aligned}
&\left|m^{n} P_{n}(1, j)-w\left(m^{-n} j\right)\right| \leqq c\left\{\mathrm{II}^{(n)}+\mathrm{I}_{2}^{(n)}+\left|\mathrm{I}_{11}^{(n)}\right|+\left|\mathrm{I}_{12}^{(n)}\right|\right\} \\
& \leqq c\left\{m^{(\alpha-1) \delta_{0} n}\left(m^{-n} j\right)^{-1}+m^{(2-3 \alpha) n}\left(m^{-n} j\right)^{-1}\right. \\
&\left.\quad+\gamma_{0}^{n}+m^{(1-2 \alpha) n}+m^{(\alpha-1) \delta_{0} n}+\gamma^{(1-\alpha) n} m^{\alpha(1-p) n}\right\}
\end{aligned}
$$

But

$$
\min _{2 / 3<\alpha<1}\left[m^{(\alpha-1) \delta_{0}} \vee m^{(2-3 \alpha)} \vee \gamma_{0}^{1-\alpha}\right]=\min _{2 / 3<\alpha<1}\left[m^{(\alpha-1) \delta_{0}} \vee m^{(2-3 \alpha)}\right]
$$

which is achieved for $\alpha=\alpha_{0}=\left(2+\delta_{0}\right) /\left(3+\delta_{0}\right)$, and then the minimum is $m^{\delta_{0} /\left(3+\delta_{0}\right)}=\beta_{0}$. 
Given any $\beta<\beta_{0}$ we can find $\gamma>\gamma_{0}$ and $0<p<1$ such that with $\alpha=\left(2+\delta_{0}\right) /\left(3+\delta_{0}\right)$ one has $\beta \gamma^{1-\alpha_{0}} m^{\alpha_{0}(1-p)}<1$. Thus the proof is complete.

REMARK. It is worth noting that the only place we use the assumption $E Z_{1}^{2}<\infty$ is to estimate

$$
I_{2}^{(n)}=\left|\frac{1}{2 \pi} \int_{0 \leqq|u| \leqq m^{(1-\alpha) n}}\left[\varphi_{n}(u)-\varphi(u)\right] \exp \left(-i j m^{-n} u\right) d u\right| .
$$

We suspect that $I_{2}^{(n)}$, like $I_{1}^{(n)}$ decays at a rate faster than $\beta^{-n}$ for any $\beta<\beta_{0}=m^{\delta_{0}}$, and that this holds under $E Z_{1} \log Z_{1}<\infty$.

5. More on $W$; the global limit law. In this section we prove the Lipschitz continuity and strict positivity of $w(\cdot)$, and the global limit law.

LEMMA 7. If $E Z_{1} \log Z_{1}<\infty$, then $w(x)$ is Lipschitz continuous of order $\delta_{0}^{\prime}=$ $\min \left(\delta_{0}, 1\right)$ for $x \geqq \varepsilon>0$ where $\varepsilon>0$ is arbitrary.

Proof. Differentiating $\varphi(u)$ we have

$$
\varphi^{\prime}(u)=i \int_{0}^{\infty} e^{i u x} x w(x) d x
$$

Now $x w(x)$ is a density function, and we know by Lemma 2 that $\varphi^{\prime}$ is integrable. Hence

$$
x w(x)=\frac{1}{2 \pi} \int_{-\infty}^{\infty} e^{-i u x} \frac{\varphi^{\prime}(u)}{i} d u
$$

and taking $y_{1}, y_{2}>0$

$$
\begin{aligned}
w\left(y_{1}\right)-w\left(y_{2}\right) & =\frac{i}{2 \pi} \int_{-\infty}^{\infty}\left\{\frac{\exp \left(-i u y_{2}\right)}{y_{2}}-\frac{\exp \left(-i u y_{1}\right)}{y_{1}}\right\} \varphi^{\prime}(u) d u \\
& =\frac{i}{2 \pi} \int_{-\infty}^{\infty} \frac{y_{1} \exp \left(-i u y_{2}\right)-y_{2} \exp \left(-i u y_{1}\right)}{y_{1} y_{2}} \varphi^{\prime}(u) d u .
\end{aligned}
$$

Adding and subtracting $y_{1}^{-1} \exp \left(-i u y_{2}\right)$, the above

$$
\begin{aligned}
= & \frac{i}{2 \pi} \frac{y_{1}-y_{2}}{y_{1} y_{2}} \int_{-\infty}^{\infty} \exp \left(-i u y_{2}\right) \varphi^{\prime}(u) d u \\
& +\frac{i}{2 \pi} \frac{1}{y_{1}} \int_{-\infty}^{\infty}\left(\exp \left(-i u y_{2}\right)-\exp \left(-i u y_{1}\right)\right) \varphi^{\prime}(u) d u \\
\equiv & L_{1}+L_{2} .
\end{aligned}
$$

Since $\varphi^{\prime}$ is integrable

$$
\left|L_{1}\right| \leqq c \frac{\left|y_{1}-y_{2}\right|}{y_{1} y_{2}}
$$

To estimate the integral in $L_{2}$, we decompose it into the ranges $A=$ $\left\{u:|u|\left|y_{2}-y_{1}\right| \leqq 1\right\}$ and $\bar{A}=$ the complement of $A$. Now for $u \in A$

$$
\frac{\left|\exp \left(-i u y_{2}\right)-\exp \left(-i u y_{1}\right)\right|}{\left|y_{2}-y_{1}\right||u|} \leqq c
$$


and hence

$$
\begin{aligned}
\int_{A}\left|\exp \left(-i u y_{2}\right)-\exp \left(-i u y_{1}\right)\right|\left|\varphi^{\prime}(u)\right| d u & \leqq c\left|y_{2}-y_{1}\right| \int_{A}|u|\left|\varphi^{\prime}(u)\right| d u \\
& =c\left|y_{2}-y_{1}\right| \int_{A}|u|^{1+\delta_{0}}\left|\varphi^{\prime}(u)\right| \frac{d u}{|u|^{\delta_{0}}} .
\end{aligned}
$$

But by Lemma $2|u|^{1+\delta_{0}}\left|\varphi^{\prime}(u)\right|$ is bounded, and hence the above

$$
\leqq c_{1}\left|y_{2}-y_{1}\right| \int_{|u| \leqq 1 /\left|y_{2}-y_{1}\right|}|u|^{-\delta_{0}} d u \leqq c_{2}\left|y_{2}-y_{1}\right|^{\delta_{0}}
$$

On the other hand

$$
\begin{aligned}
\int_{A}\left|\exp \left(-i u y_{2}\right)-\exp \left(-i u y_{1}\right)\right|\left|\varphi^{\prime}(u)\right| d u & \leqq 2 \int_{A}\left|\varphi^{\prime}(u)\right| d u \\
& \leqq c \int_{A} \frac{d u}{|u|^{1+\delta_{0}}} \leqq c_{1}\left|y_{2}-y_{1}\right|^{\delta_{0}}
\end{aligned}
$$

(by Lemma 2).

Combining (2), (3), and (4) with (1) yields

$$
\left|w\left(y_{1}\right)-w\left(y_{2}\right)\right| \leqq c^{\prime}\left|y_{1}-y_{2}\right| / y_{1} y_{2}+c^{\prime \prime}\left|y_{1}-y_{2}\right|^{\delta_{0}} / y_{1},
$$

which implies the lemma.

One can also quite easily prove the following:

Lemma 8. Let $\delta_{0}>1$. Then $w(x)$ is differentiable at least $p$ times, where $p$ is the greatest integer strictly less than $\delta_{0}$.

This lemma is meaningful only in the discrete case since $\delta_{0} \leqq 1$ in the continuous case. However, Karlin [8] has shown that in the continuous case $w(x)$ is infinitely differentiable.

From Lemma 7 and Theorem 1 we easily get the sharp form of the global limit law. We again make the simplifying assumption $q=0$. We shall prove the following form of Theorem 2:

THEOREM 2a. Fix $0<x_{1}<x_{2}<\infty$. Then under the hypothesis of Theorem 2 we can, for any $\beta<\beta_{0}$, find a constant $C=C(\beta)$ such that

$$
\left|P\left\{x_{1}<W_{n} \leqq x_{2}\right\}-P\left\{x_{1}<W \leqq x_{2}\right\}\right| \leqq C \beta^{-n} .
$$

Proof. As before we assume without loss of generality that $P\left(Z_{0}=1\right)=1$.

$$
\begin{aligned}
\mid P\left\{x_{1}<W_{n} \leqq x_{2}\right\} & -P\left\{x_{1}<W \leqq x_{2}\right\} \mid \\
\leqq & \left|m^{-n} \sum_{j=x_{1} m^{n}}^{x_{2} m^{n}}\left\{m^{n} P_{n}(1, j)-w\left(m^{-n} j\right)\right\}\right| \\
& \quad+\left|\sum_{j=x_{1} m^{n}}^{x_{2} m^{n}} m^{-n} w\left(m^{-n} j\right)-\int_{x_{1}}^{x_{2}} w(x) d x\right| \\
= & \left|J_{1}^{(n)}\right|+\left|J_{2}^{(n)}\right| .
\end{aligned}
$$


By Theorem 1a, each term in the first sum is $o\left(\beta^{n}\right)$ uniformly for $j$ in the range of summation. The number of terms is $\left(x_{2}-x_{1}\right) m^{n}$, and hence

$$
J_{1}^{(n)}=o\left(\beta^{n}\right) \text {. }
$$

Next we write $J_{2}^{(n)}$ in the form

$$
\left|J_{2}^{(n)}\right|=\left|\sum_{x_{1} \leqq j m^{-n} \leqq x_{2}} \int_{j^{-n}}^{(j+1) m^{-n}}\left[w(x)-w\left(m^{-n} j\right)\right] d x\right| .
$$

But according to Lemma 7 (see end of proof) $w(\cdot)$ is Lipschitz of order $\delta_{0}^{\prime}$, i.e.

$$
|w(x)-w(y)| \leqq K|x-y|^{\delta_{0}^{\prime}} \text { for } x, y \geqq \varepsilon>0 .
$$

Thus

$$
\begin{aligned}
\left|J_{2}^{(n)}\right| & \leqq K \sum \int_{j m^{-n}}^{(j+1) m^{-n}}\left|x-m^{-n} j\right|^{\delta_{0}^{\prime}} d x=K \sum_{j=m^{n} x_{1}}^{m^{n} x_{2}} \int_{0}^{m-n} y^{\delta_{0}^{\prime}} d y \\
& =\frac{1}{1+\delta_{0}^{\prime}} K m^{n}\left(x_{2}-x_{1}\right) m^{-n\left(1+\delta_{0}^{\prime}\right)}=K_{1}\left(x_{2}-x_{1}\right) m^{-\delta_{0}^{\prime} n} .
\end{aligned}
$$

But $\beta<\beta_{0}<m^{\delta_{0}}$ and this proves the theorem.

THEOREM 3. If $E Z_{1} \log Z_{1} \leq \underline{\infty}$, then the density $w(x)$ is strictly positive for all $x>0$.

Proof. By Lemma 2, $\left|\varphi^{\prime}\right|$ is integrable. Thus $x w(x)$ and hence $w(x)$ is continuous for $x>0$. Since there exists at least one point where $w(\cdot)>0$, there is also an open interval $I=(a, b)$ such that $w(x)>0$ for $x \in I$. Since $m>1$ there must be integers $k_{1}, k_{2}$, such that $1 \leqq k_{1}<m<k_{2}$ and such that $P\left(1, k_{1}\right)>0$ and $P\left(1, k_{2}\right)>0$. Fix $k_{1}$ and $k_{2}$ for the remainder of the proof.

Using again the identity

$$
\varphi(m u)=f[\varphi(u)]
$$

we readily deduce that

$$
w^{(i)}(x)=\sum_{j} P(i, j) m w^{(j)}(m x),
$$

where $w^{(i)}$ denotes the $n$-fold convolution of $w$. Since $P\left(1, k_{1}\right)>0$ and $P\left(1, k_{2}\right)>0$ we see from (10) that

$$
w^{(i)}(x) \geqq c w^{\left(i k_{1}\right)}(m x), \quad i=1,2, \ldots,
$$

and

$$
w^{(i)}(x) \geqq c w^{\left(i k_{2}\right)}(m x), \quad i=1,2, \ldots .
$$

Now suppose $w^{(i)}(x)>0$ for $x \in J$ where $J$ is some set of real numbers. Then $w^{\left(i k_{2}\right)}(x)>0$ for $x \in k_{2} J$, which by (12) implies that $w^{(i)}(x)>0$ for $x \in\left(k_{2} / m\right) J$. Repeating this argument $n$ times we see that

$$
w^{(i)}(x)>0 \text { for } x \in\left(k_{2} / m\right)^{n} J, \text { for all } n \geq 0 \text {. }
$$


Therefore $w^{\left(i k_{1}\right)}(x)>0$ for $x \in k_{1}\left(k_{2} / m\right) J$ and applying (11) we see that $w^{(i)}(x / m)>0$ for $x \in k_{1}\left(k_{2} / m\right)^{n} J$, or $w^{(i)}(x)>0$ for $x \in\left(k_{1} / m\right)\left(k_{2} / m\right)^{n} J$. Repeating the latter step $N$ times we conclude that $w(x)>0$ for $x \in J$ implies

$$
w^{(i)}(x)>0 \text { for } x \in\left(k_{1} / m\right)^{N}\left(k_{2} / m\right)^{n} J, \quad n, N \geqq 0 .
$$

In particular since $w(x)>0$ for $x \in I$, we see from (14) with $i=1$ and $J=I$ that

$$
w(x)>0 \text { for } x \in S \equiv \bigcup_{n, N \geq 0}\left\{\left(\frac{k_{1}}{m}\right)^{N}\left(\frac{k_{2}}{m}\right)^{n} I\right\}
$$

To complete the proof we use repeatedly the following fact. If $u(x)$ and $v(x)$ are densities on the line, with $u(x)>0$ for $x \in A$ and $v(x)>0$ for $x \in B$ ( $A, B$ being subsets of the reals), then

$$
(u * v)(x)>0 \text { for } x \in A+B=\{y+z: y \in A, z \in B\} .
$$

Now from the definition of $S$ in (15) and the fact that $\left(k_{1} / m\right)<1$ there is a sequence of intervals $\left\{I_{n}\right\}=\left\{\left(a_{n}, b_{n}\right)\right\} \subset S$ such that $a_{n} \rightarrow 0, b_{n} \rightarrow 0$ as $n \rightarrow \infty$, and from (16)

$$
w^{(2)}(x)>0 \text { for } x \in I+I_{n}=\left(a+a_{n}, b+b_{n}\right)
$$

for $n=1,2, \ldots$ Since $a_{n} \rightarrow 0$ we conclude that

$$
w^{(2)}(x)>0 \text { for } x \in(a, b),
$$

and also we note that we can choose an $n_{0}$ so that $a+a_{n_{0}}<b$. Hence (17) and (18) imply

$$
w^{(2)}(x)>0 \text { on }\left(a, b+b_{n_{0}}\right) \text {. }
$$

Applying the same reasoning to $w^{(3)}(x)$ we get

$$
\begin{aligned}
& w^{(3)}(x)>0 \text { on }\left(a, b+b_{n_{0}}\right)+I_{n}=\left(a+a_{n}, b+b_{n_{0}}+b_{n}\right) \text {, } \\
& \Rightarrow w^{(3)}(x)>0 \text { on }\left(a, b+b_{n_{0}}\right) \text {, } \\
& \Rightarrow w^{(3)}(x)>0 \text { on }\left(a+b_{n_{0}}, b+b_{n_{0}}+b_{n_{0}}\right) \text {, } \\
& \Rightarrow w^{(3)}(x)>0 \text { on }\left(a+2 b_{n_{0}}\right) \text {. }
\end{aligned}
$$

Continuing in this fashion we see that given any $d<\infty$, there is a $K_{0}$ such that for any integer $k_{0}>K_{0}$

$$
w^{\left(k_{0}\right)}(x)>0 \text { on }(a, d) .
$$

Choose $d$ so that $k_{2} / m<d / a$. Then $\bigcup_{n=0}^{\infty}\left(k_{2} / m\right)^{n}(a, d)=(a, \infty)$, and hence applying (14) with $i=k_{0}, N=0$ and $J=(a, d)$, we see that

$$
w^{\left(k_{0}\right)}(x)>0 \text { for } x \in(a, \infty) \text {. }
$$

Reapplying (21) to (14) with $i=k_{0}, n=0$ and $J=(a, \infty)$ we see that

$$
w^{\left(k_{0}\right)}(x)>0 \text { for } x \in\left(k_{1} / m\right)^{N}(a, \infty), \quad N=0,1, \ldots,
$$


or, since $\left(k_{1} / m\right)<1$,

$$
w^{\left(k_{0}\right)}(x)>0 \text { for } x>0, \quad k_{0} \geqq K_{0} .
$$

Now (12) with $i=1$ implies

$$
w(x) \geqq c w^{\left(k_{2}\right)}(m x) \geqq c^{2} w^{\left(k_{2}^{r}\right)}\left(m^{2} x\right) \geqq \cdots \geqq c^{r} w^{\left(k_{2}^{r}\right)}\left(m^{r} x\right), \quad r \geqq 1 .
$$

Choose $k_{0} \geqq K_{0}$ so that $k_{0}=k_{2}^{r}$ for some integer $r$. (This is possible since $k_{2}>1$.) Then (22) and (23) imply

$$
w\left(x / m^{r}\right)=w^{\left(k_{0} / k_{2}^{r}\right)}\left(x / m^{r}\right) \geqq c^{r} w^{\left(k_{0}\right)}(x)>0 \text { for } x>0 .
$$

Hence $w(x)>0$ for $x>0$.

\section{Some further observations.}

6.1. The space-time boundary. If we let $\mathscr{P}_{n}\left[\left(i_{1}, n_{1}\right),\left(i_{2}, n_{2}\right)\right]$ denote the $n$-step transition function of the space-time process, then the Green function (renewal function) for this process is

$$
G\left[\left(i_{1}, n_{1}\right),\left(i_{2}, n_{2}\right)\right]=\sum_{n} \mathscr{P}_{n}\left[\left(i_{1}, n_{1}\right),\left(i_{2}, n_{2}\right)\right]=P_{n_{2}-n_{1}}\left(i_{1}, i_{2}\right),
$$

where $P_{n}(i, j)$ is the transition function for the original Galton-Watson process. Fix $\left(i_{0}, n_{0}\right)$. We want to find

$$
\lim _{(j, N) \rightarrow \infty} \frac{G[(i, n),(j, N)]}{G\left[\left(i_{0}, n_{0}\right),(j, N)\right]}=\lim _{(j, N) \rightarrow \infty} \frac{P_{N-n}(i, j)}{P_{N-n_{0}}\left(i_{0}, j\right)}=h(i, n)
$$

for all possible sequences $(j, N) \rightarrow \infty$. Any harmonic function (i.e. a function $g(\cdot, \cdot)$ satisfying $g=\mathscr{P} g$ ) can then be expressed as a mixture (with respect to some measure) of the functions $h$ obtained as limits in (2). (For a general discussion of these matters see e.g. Kemeny, Snell and Knapp [9].)

Now if $(j, N) \rightarrow \infty$ in such a way that $j m^{-N} \rightarrow x, 0<x<\infty$, then we can use Theorem 1 to evaluate the limits in (2). (This observation has been known to many workers-e.g. S. Karlin, L. Snell-and we make no claim of originality here.) Theorem 1 yields

$$
\lim _{j m^{-n} \rightarrow x} m^{n} P_{n}(i, j)=w^{(i)}(x)
$$

Using this fact we have

$$
\begin{aligned}
\lim _{(j, N) \rightarrow \infty ; j m-N \rightarrow x} \frac{P_{N-n}(i, j)}{P_{N-n_{0}}\left(i_{0}, j\right)} & =\lim m^{n-n_{0}} \cdot \frac{m^{N-n} P_{N-n}(i, j)}{m^{N-n_{0}} P_{N-n_{0}}\left(i_{0}, j\right)} \\
& =m^{n-n_{0}} \frac{w^{(i)}\left(x m^{n}\right)}{w^{\left(i_{0}\right)}\left(x m^{n_{0}}\right)}=c_{x} m^{n} w^{(i)}\left(x m^{n}\right),
\end{aligned}
$$

where the constant $c_{x}$ depends on $i_{0}, n_{0}$, and $x$. Let

$$
h_{x}(i, n)=c_{x} m^{n} w^{(i)}\left(x m^{n}\right) .
$$


The functions $h_{x}$ are easily seen to be harmonic functions, namely to satisfy

$$
h_{x}(i, n)=\sum_{(j, N)} \mathscr{P}[(i, n),(j, N)] h(j, N)=\sum_{j} P(i, j) h_{x}(j, n+1),
$$

i.e.

$$
w^{(i)}(x)=\sum_{j} P(i, j) m w^{(i)}(m x)
$$

which is precisely (5.10).

At the moment we do not know what the other limits are. However, we can say the following. Suppose that there were a sequence $\left(j_{k}, N_{k}\right)$ such that

$$
\lim _{\left(j_{k}, N_{k}\right) \rightarrow \infty} \frac{P_{N_{k}-n}\left(i, j_{k}\right)}{P_{N_{k}-n_{0}}\left(i_{0}, j_{k}\right)}=h(i, n) \text { exists for all }(i, n),
$$

but $h(i, n) \equiv h_{x}(i, n)$ for any of the functions given in (5). Let $\alpha_{k}=j_{k} m^{-N_{k}}$. If $\alpha_{k} \rightarrow 0$ or $\infty$ then there must be a subsequence $\alpha_{k^{\prime}}$ such that $\alpha_{k^{\prime}} \rightarrow \alpha \in(0, \infty)$. Thus

$$
\frac{P_{N_{k^{\prime}}-n}\left(i, j_{k^{\prime}}\right)}{P_{N_{k^{\prime}}-n_{0}}\left(i, j_{k^{\prime}}\right)} \rightarrow h_{\alpha}(i, n),
$$

and hence the same must be true for the original sequence. Hence if there are any limits other than those in (5) they must be limits of ratios of the form (8) where $\alpha_{k} \rightarrow 0$ or $\infty$.

When the process is embeddable in continuous time one can use the following result, claimed by Karlin [8], to conclude that if $\alpha_{k} \rightarrow 0$ then $h(i, n)=1$ for all $(i, n)$.

THEOREM (KARLIN). Under the notation and condition of Theorem 1 one has, for any $i$,

$$
\lim _{(j, n) \rightarrow \infty: m^{-n_{j} \rightarrow 0}} m^{n}\left(m^{-n} j\right)^{1-\delta_{0}} P_{n}(i, j)=b \quad \text { where } 0<b<\infty .
$$

Even in the embeddable situation the case when $\alpha_{k} \rightarrow \infty$ is not settled yet. These questions are under study. The behavior of $w(x)$ near the origin and $\infty$ will be essential in this. We have a few partial results in this direction as given below.

6.2. Behavior of $w(\cdot)$ near zero. In his 1948 paper [4], T. Harris gave some partial results on the behavior of $F(x)=P\{W \leqq x\}$ near $x=0$. He proved a Tauberian type theorem, in which an average of $F$ was described, namely

$$
\lim _{\alpha \rightarrow 0} \int_{x}^{\alpha x} v^{-\delta_{0}-1} F(v) d v=\frac{1}{\Gamma\left(\delta_{0}+1\right)} \int_{1}^{x} \frac{M(v)}{v} d v,
$$

where $M(s)$ was a function satisfying

$$
M(s) \sim s^{\delta_{0}} \varphi(i s), \quad s \rightarrow \infty ; \quad M(m s)=M(s) .
$$

We wish to observe here that if one restricts oneself to sequences $\left\{x_{n}, n=0,1, \ldots\right\}$ of the form $x_{n}=x m^{-n}, 0<x<\infty$, then one can describe the asymptotics of $F\left(x_{n}\right)$ 
itself; and under further restrictions, that of $w\left(x_{n}\right)$. Namely, applying the Karamata Tauberian theorem [14] to Lemma 2(i) (note that the latter holds for Laplace transforms as well) we get

$$
\left.F\left(m^{-k} x\right) \sim \frac{A[\varphi(i / x)]}{\Gamma\left(\delta_{0}+1\right)} \gamma_{0}^{k} \quad \text { (here } A(s) \text { is as in Lemma } 1\right) .
$$

By Lemma 8 we see that if $\delta_{0}>1$ then $w(\cdot)$ is differentiable and

$$
\begin{aligned}
w^{\prime}(x) & =\frac{1}{2 \pi x^{2}} \int_{-\infty}^{\infty} e^{-i u x} \varphi^{\prime}(u) d u+\frac{i}{2 \pi x} \int_{-\infty}^{\infty} e^{-i u x} u \varphi^{\prime}(u) d u \\
& =\frac{1}{2 \pi x^{3}} \int_{-\infty}^{\infty} e^{-i v} \varphi^{\prime}\left(\frac{v}{x}\right) d v+\frac{i}{2 \pi x^{2}} \int e^{-i v}\left(\frac{v}{x}\right) \varphi^{\prime}\left(\frac{v}{x}\right) d v \\
& =O\left(x^{\delta_{0}-2}\right) \quad \text { as } x \rightarrow 0 .
\end{aligned}
$$

We can therefore apply Corollary 4.4a, p. 194 of Widder [14], and conclude that

$$
w\left(m^{-k} x\right) \sim\left(A[\varphi(i / x)] / x \Gamma\left(\delta_{0}\right)\right) m^{-k\left(\delta_{0}-1\right)} .
$$

Since $\delta_{0}=\left(\log \left(1 / p_{1}\right)\right) / \log m$ we see that (2) will hold if $p_{1}$ is sufficiently small. If $p_{1}$ is not sufficiently small then (1) and (2) suggest that $w(y)$ blows up at the rate $(1 / y)^{1-\delta_{0}}$, but also oscillates wildly in the process.

In the continuous case Karlin [8] has shown that actually $w(y) \sim$ const $y^{\delta_{0}-1}$ as $y \rightarrow 0$, and the density thus does not oscillate in this case.

6.3. Acknowledgements. After this paper was completed the authors were informed (by A. Joffe) of the beautiful work of S. Dubuc on boundary theory for supercritical Galton-Watson branching process. By an ingenious trick Dubuc establishes the following form of local limit law just under the assumption that $E Z_{1}\left(\log Z_{1}\right)<\infty$ and using nothing more than the dominated convergence theorem: Let $j^{\prime}$ se a sequence of integers going to infinity such that $\lim _{n \rightarrow \infty} j_{n} m^{-n}=c$ where $0<c<\infty$. Then $j_{n} P_{n}(i, j) \rightarrow c w(c)$.

He does not, however, establish any rates of convergence. There is some further overlap between Dubuc's paper and ours. He too establishes the strict positivity of $w(x)$ using essentially the same method. We understand from S. Karlin that S. Watanabe also showed a proof as early as 1965 .

Dubuc's paper will appear in Studia Mathematica and is entitled La fonction de Green d'un processes du Galton-Watson."

We would also like to thank the referee for several useful observations and for pointing out an error in an earlier version of Theorem 3.

\section{REFERENCES}

1. K. B. Athreya and P. Ney, Branching processes, (to appear 1970).

2. V. P. Cistyakov, Local limit theorems for branching processes, Teor. Verojatnost. i Primenen 2 (1957), 360-374= Theor. Probability Appl. 2 (1957), 345-364. MR 19, 1092.

3. G. H. Hardy and E. M. Wright, An introduction to the theory of numbers, Clarendon Press, Oxford, 1938. 
4. T. E. Harris, Branching processes, Ann. Math. Statist. 19 (1948), 474-494. MR 10, 311; 856.

5. - The theory of branching processes, Die Grundlehren der Math. Wissenschaften, Band 119, Springer-Verlag, Berlin, 1963. MR 29 \#664.

6. D. Hawkins and S. Ulam, Theory of multiplicative processes, Los Alamos Scientific Laboratory, 1944, LADC-265.

7. Haruo Imai, Notes on a local limit theorem for discrete time Galton-Watson branching processes, Ann. Inst. Statist. Math. 20 (1968), 391-410. MR 38 \#6678.

8. S. Karlin, Local limit laws for the supercritical continuous time simple branching processes, 1967 (unpublished manuscript).

9. J. Kemeny, L. Snell and A. Knapp, Denumerable Markov chains, Van Nostrand, Princeton, N. J., 1966. MR 34 \#6858.

10. H. Kesten, P. Ney and F. Spitzer, The Galton-Watson process with mean one and finite variance, Teor. Verojatnost. i Primenen. 11 (1966), 579-611. MR 34 \#6868.

11. H. Kesten and B. P. Stigum, A limit theorem for multidimensional Galton-Watson processes, Ann. Math. Statist. 37 (1966), 1211-1223. MR 33 \#6707.

12. E. Seneta, Functional equations and the Galton-Watson process, Advances Appl. Probability 1 (1969), 1-42.

13. B. P. Stigum, A theorem on the Galton-Watson process, Ann. Math. Statist. 37 (1966), 695-698. MR 33 \#4996.

14. David Vernon Widder, The Laplace transform, Princeton Math. Series, vol. 6, Princeton Univ. Press, Princeton, N. J., 1941. MR 3, 232.

15. A. M. Yaglom, Certain limit theorems of the theory of branching random processes, Dokl. Akad. Nauk SSSR 56 (1947), 795-798. (Russian) MR 9, 149.

UNIVERSITY OF WISCONSIN, MADISON, Wisconsin 53706 